\title{
DUAL SYMMETRY OF PROJECTIVE SETS IN A FINITE MODULAR LATTICE
}

\author{
BY \\ S. P. AVANN
}

1. Introduction. In order to motivate the principal result of this paper we review here some elementary theory in a finite modular and distributive lattice.

THEOREM 1.1. In a finite modular lattice $L$ an element $y$ is a join irreducible if and only if it covers exactly one element $b \in L$ and if and only if $y / b$ is a minimal prime quotient (has no lower transposed quotients). And dually, $x$ is a meet irreducible if and only if it is covered by exactly one element $a \in L$ and if and only if $a / x$ is a maximal prime quotient.

THEOREM 1.2. In a finite distributive lattice $L$ the partially ordered set $P$ of join irreducibles $y$ is isomorphic to the partially ordered set $P^{\prime}$ of meet irreducibles $x$, where ordering in $P$ and $P^{\prime}$ is the ordering of $L$. The correspondence $y \leftrightarrow x$ between $P$ and $P^{\prime}$ may be given by any one of the following characterizations:

$$
y=\bigcap_{a_{i} \nsupseteq^{x}} a_{i} ; \quad x=\bigcup_{b_{i} \nsupseteq y} b_{i} .
$$

(ii) The dual ideal $u / y$ and the ideal $x / z$ are complementary sets in $L$.

(iii) $y / b$, where $y>b$ ( $y$ covers $b)$, is the unique minimal prime quotient, and $a / x$, where $a>x$ is the unique maximal prime quotient in the same complete set $Q$ of projective prime quotients.

We shall obtain the following generalization of property (iii) above for the modular case:

In each complete set $Q$ of projective prime quotients of a finite modular lattice $L$ the minimal prime quotients of $Q$ may be paired off in a 1-1 (not necessarily unique) manner with the maximal prime quotients of $Q$. By Theorem 1.1 this gives a 1-1 correspondence between the join irreducibles and the meet irreducibles associated with each complete set $Q$ of projective prime quotients of $L$.

We shall generalize this result further in two directions: (1) $Q$ may be taken as the complete set of quotient (convex) sublattices projective with an arbitrary complemented quotient sublattice of $L$; and (2) the 1-1 pairing of quotients of $Q$ is essentially extended to all the members of $Q$ rather than merely to the minimal and maximal quotients. To clarify these statements we return again to the distributive case for further properties.

Presented to the Society May 1, 1954; received by the editors March 4, 1957. 
THEOREm 1.3. A finite distributive lattice $L$ is isomorphic to the point set algebra of the $M$-closed $[1, \mathrm{p} .14]$ subsets of the partially ordered set $P$ of its join irreducible elements under the correspondence $b \leftrightarrow B$ where $B$ is the set of join irreducibles contained in $b$. Dually, $L$ is dually isomorphic to the point set algebra of $J$-closed [1, p. 14] subsets of the partially ordered set $P^{\prime}$ of its meet irreducible elements under the correspondence $a \leftrightarrow A$, where $A$ is the set of meet irreducibles containing $a$.

THEOREM 1.4. In a finite distributive lattice $L$ an element $b$ covers exactly $r$ elements $b_{1}, b_{2}, \cdots, b_{r}$ if and only if there are $r$ join irreducibles $y_{1}, y_{2}, \cdots, y_{r}$ in the reduced (irredundant) join irreducible representation of $b$. The latter are the maximal join irreducibles of $B$, the set of all join irreducibles contained in $b$ in the isomorphism of Theorem 1.3. Furthermore, $b_{1}, b_{2}, \cdots, b_{r}$ generate under $b$ the quotient sublattice $b / b_{*}, b_{*}=b_{1} \cap \ldots \cap b_{r}$, which is a Boolean algebra $\mathbb{B}^{r}$.

THEOREM 1.5. In a finite distributive lattice $L$ there exists a 1-1 correspondence between the elements of $L$ and themselves determined uniquely as follow:

$$
y_{1} \cup \cdots \cup y_{r}=b \leftrightarrow a=x_{1} \cap \cdots \cap x_{r}
$$

where $y_{1} \cup \ldots \cup y_{r}$ is the reduced join irreducible representation of $b$, $x_{1} \cap \cdots \cap x_{r}$ is the reduced meet irreducible representation of $a$, and $y_{i}$ and $x_{i}$ are corresponding join and meet irreducibles $(i=1,2, \cdots, r)$ in the isomorphism $P \simeq P^{\prime}$ of Theorem 1.2. Furthermore the quotient sublattices $b / b_{*}$ and $a^{*} / a$ of Theorem 1.4 and its dual are respectively the unique minimal and maximal quotients of a complete set of projective quotient sublattices, each complemented and distributive, hence a Boolean algebra $\mathrm{B}^{r}$.

The essential parts of the proofs of Theorems 1.1-1.5 are found in Birkhoff's Lattice theory [1] and the details are left to the reader.

Our generalization of Theorem 1.5 to a modular lattice states that each complete set $Q$ of projective complemented quotient sublattices has the same number of minimal and maximal quotients. These are respectively of the form $b / b_{*}$, the complemented $[1$, p. 114 , Corollary to Theorem 1 and its dual] quotient sublattice generated under $b$ by the elements covered by $b$, and $a^{*} / a$ the complemented [ibid.] quotient sublattice generated above $a$ by the elements covering $a$. We shall obtain this result as a special case from the principal theorem to which we now turn.

\section{The principal theorem.}

THEOREM 2A. Let $Q_{0}, Q_{1}, \cdots, Q_{r}$ constitute all of the distinct complete sets of projective complemented quotient sublattices of a finite modular lattice $L$ and let $k_{0}, k_{1}, \cdots, k_{r}$ be an arbitrary fixed set of non-negative integers. Let $V\left(k_{0}, k_{1}, \cdots, k_{r}\right)$ be the set of all elements $x$ for which there are precisely $k_{i}$ quotients belonging to $Q_{i},(i=0,1, \cdots, r)$ that are minimal in the complemented cuotient sublattice $x^{*} / x$ generated above $x$ by the elements covering $x$. 
Dually, let $W\left(k_{0}, k_{1}, \cdots, k_{r}\right)$ be the set of all elements $y$ for which there are precisely $k_{i}$ quotients belonging to $Q_{i},(i=0,1, \cdots, r)$, that are maximal in the complemented quotient sublattice $y / y_{*}$ generated under $y$ by the elements covered by $y$. Then, the orders of $V$ and $W$ are equal.

We note that a complemented quotient $a / b$ in the complemented quotient sublattice $x^{*} / x$ is minimal if and only if $b=x$. For otherwise $b^{\prime} / x$ would be a proper lower transpose of $a / b$ in $x^{*} / x$, where $b^{\prime}$ is the relative complement of $b$ in $a / x$. We note also that every $x \in L$ must fall into one and only one nonempty set $V$ and every $y \in L$ must fall into one and only one nonempty set $W$. In view of these two facts we can state Theorem $2 \mathrm{~A}$ in the alternate form:

THEOREM 2B. There exists a one-one correspondence of elements of a finite modular lattice $L$ with themselves: $x \leftrightarrow y$, such that $x$ will be the denominator of the same number of quotients from $Q_{i}$ as the number of quotients from $Q_{i}$ that have $y$ as their numerator $(i=0,1, \cdots, r)$.

The author conjectured the validity of this theorem in 1940. R. P. Dilworth proved in [3] a quite specialized version of this result by applying the abstract Möbius function, which was first applied to partially ordered sets by Weisner in [5].

A discussion of the special cases, including Dilworth's result, will follow the proof of the theorem. However, a few remarks should precede it.

The quotients from one set $Q_{i}$ may be found as sub-quotient lattices from another set $Q_{j}$. Then, $k_{j}>0$ requires $k_{i}>0$ for nonempty sets $V$ and $W$.

Let $Q_{0}$ be the set of trivial quotients of length $0, V(1,0, \cdots, 0)$ consists of the unit element $u$ only and $W(1,0, \cdots, 0)$ consists of the zero element $z$ only. Elements $u$ and $z$ are the only elements of $L$ with no structure above and no structure below, respectively.

Since every $x \in L$ is the denominator of a unique number of complemented quotient sublattices from each complete set $Q_{i},(i=0,1, \cdots, r)$, the set of nonempty $V\left(k_{0}, k_{1}, \cdots, k_{r}\right)$ is a partitioning of $L$ as a set into nonintersecting subsets of $x$ 's. Dually the set of all nonempty $W\left(k_{0}, k_{1}, \cdots, k_{r}\right)$ is a partitioning of $L$ into nonintersecting subsets of $y$ 's.

\section{3 . The complemented case.}

When $L$ is complemented we could prove the theorem readily by a duality argument, if it were not for the possible presence of non-Desarguesian finite projective planes. These generally do not admit a dual automorphism, whereas all other finite projective geometries and direct products thereof do. We shall not necessarily apply the duality argument in the simpler cases in favor of giving more numerical detail on the $V$ sets and $W$ sets. It will be convenient hereafter to let $Q_{0}$ always be the set of trivial zero-length quotients, namely the set of the elements of $L$ themselves considered as quotients. We shall always then have $V(1,0, \cdots, 0)=u$ and $W(1,0, \cdots, 0)=z$. 
Lemma 3.1. Theorem 2 is true for the trivial one element lattice $u=z$.

Here $Q_{0}=u / z=u / u$ and $V(1)=W(1)=u$.

Lemma 3.2. Theorem 2 is true for ihe two element lattice $B^{1}$.

Here $Q_{1}=u / z, V(1,0)=u=W(1,1)$, and $V(1,1)=z=W(1,0)$.

Lemma 3.3. Theorem 2 is true for the projective line consisting of $n+1$ points for arbitrary $n>1$.

This case includes the so-called exceptional projective lines wherein $n$ fails to be a prime power $p^{s}$. The complete complemented projective sets $Q_{0}, Q_{1}, Q_{2}$ are the sets of all quotients of lengths $0,1,2$ respectively. We classify all the elements of $L$ as $x$ 's and as $y$ 's:

$$
\begin{array}{cll}
u & V(1,0,0) & W(1, n+1,1) \\
\text { the } n+1 \text { points } & V(1,1,0) & W(1,1,0) \\
z & V(1, n+1,1) & W(1,0,0)
\end{array}
$$

All other $V$ sets and $W$ sets are empty.

Lemma 3.4. Theorem 2 is true for an arbitrary non-Desarguesian finite projective plane geometry.

Proof. We first review a proof that

(a) The number of points on a line is equal to the number of lines on a point. Furthermore, letting $n+1$ be this number, $n+1>2$.

(b) The number of points and the number of lines are both equal to $n^{2}+n+1$.

If $x$ is a line, an element of rank 2 , and $x^{\prime}$ is a complement, then $u / x^{\prime}$ is an upper transpose of $x / z$. Then by Theorem 7 [1, p. 121], its dual, and the transitivity of projectivity, all quotients of length 2 are projective. They are furthermore isomorphic quotient sublattices by Theorem 6 [1, p. 73]. Hence the first statement of (a) follows. That $n+1>2$, is a postulate for a projective geometry [2, p. 323]. Let $M_{1}$ be the number of points and $M_{2}$ be the number of lines. Then $M_{1} \cdot(n+1)$ is the number of incidences of points lying on a line, and $M_{2} \cdot(n+1)$ is the number of incidences of lines lying on a point. Equality of these products yields $M_{1}=M_{2}=M$, say. To evaluate $M$, consider a fixed line $x$ and the distinct $n+1$ points $q_{0}, q_{1}, \cdots, q_{n}$ it contains. Each $q_{i}$ is contained in precisely $n$ other lines $x_{i 1}, \cdots, x_{i n}$ distinct from $x$ and from each other $(i=0,1, \cdots, n)$. For assume $x_{i r}=x_{j s}$ with $i \neq j$. Then $q_{i}<x_{i r}=x_{j s}>q_{j} \neq q_{i}$; whence $x_{i r}=q_{i} \cup q_{j}=x$, contradicting our selection of the $x_{i r}$. Finally, since every line of $L$ other than $x$ intersects $x$ in a point necessarily one of the $q_{i}$, we have accounted for all the $M$ lines. Hence, $M=1+n$ $\cdot(n+1)$.

For all examples known, $n$ is a prime power $p^{s}$, though this has never been 
proved to be necessary. A little is known about $n$. For example, $n \not \equiv 1,2 \bmod 4$ if $n \neq a^{2}+b^{2}$.

The complete sets of projective complemented quotients are, $Q_{0}, Q_{1}, Q_{2}, Q_{3}$, the sets of all quotients of length $0,1,2,3$ respectively. We classify below all the elements of $L$ as $x$ 's and $y$ 's.

$\begin{array}{cll}u & V(1,0,0,0) & W\left(1, n^{2}+n+1, n^{2}+n+1,1\right) \\ \text { The }\left(n^{2}+n+1\right) \text { lines } & V(1,1,0,0) & W(1, n+1,1,0) \\ \text { The }\left(n^{2}+n+1\right) \text { points } & V(1, n+1,1,0) & W(1,1,0,0) \\ z & V\left(1, n^{2}+n+1, n^{2}+n+1,1\right) & W(1,0,0,0)\end{array}$

All other $V$ and $W$ sets are empty. As will be true for every complemented case, the set of $y$ 's corresponding to a set of $x$ 's will be the set of complements of the $x$ 's.

Lemma 3.5. Theorem 2 is true for an arbitrary finite projective geometry $\mathrm{PG}\left(m, p^{s}\right), m \geqq 2, p^{s}$ an arbitrary prime power, where $\mathrm{PG}\left(m, p^{s}\right)$ is the lattice of all subspaces of the $(m+1)$-dimensional vector space of $(m+1)$-vectors with homogeneous coordinates from the finite division ring $\mathrm{GF}\left(p^{s}\right)$. For $m=2$ this case includes all finite Desarguesian projective planes.

Proof. We show first that all quotient sublattices of the same lattice rank $h+1$, i.e. of dimension $h$, are projective. Let $c / b$ be an arbitrary quotient lattice of length $h+1$ in $L=\operatorname{PG}\left(m, p^{s}\right)$. By Theorem 1 of Birkhoff $[1$, p. 114] $b$ has a relative complement $b^{\prime}$ in $c / z$, making $c / b$ an upper transpose of $b^{\prime} / z$. These transposed quotients are isomorphic and therefore of same length (rank) and dimension by Theorem 6, Birkhoff $[1$, p. 73]. But by Theorem 7 $[1$, p. 121] all $d / z$ of the same length are projective and moreover isomorphic to $\mathrm{PG}\left(h, p^{s}\right)[2, \mathrm{p} .327]$. By the Wedderburn Theorem the coordinate field $\mathrm{GF}\left(p^{s}\right)$, since finite, is commutative and is trivially anti-isomorphic with itself. By Theorem $9[1$, p. 123$]$ this guarantees that $\operatorname{PG}\left(m, p^{s}\right)$ has a dual automorphism. The projective set of all $m$-dimensional quotient lattices, each possessing dual automorphisms, is carried into itself by a dual automorphism of $L=\mathrm{PG}\left(m, p^{s}\right)$. Therefore, Theorem 2 is valid by duality, since the $V$ and $W$ sets are duals of one another, completing the proof. Again we note that a $W$ set is the set of all complements of the corresponding $V$ set.

We now set forth to describe the nonempty $V$ and $W$ sets. The complete sets of projective complemented quotient sublattices of $\mathrm{PG}\left(m, p^{s}\right)$ are $Q_{0}, Q_{1}, \cdots, Q_{m+1}$, where $Q_{h+1}$ is the set of all quotient sublattices of dimension $h$, each of length $h+1$ and isomorphic to $\operatorname{PG}\left(h, p^{s}\right),(h=-1,0,1, \cdots, m)$. Here $\operatorname{PG}\left(0, p^{s}\right)$ can be defined to be $B^{1}$, the two element Boolean algebra and $\mathrm{PG}\left(-1, p^{s}\right)$ the one element lattice $B^{0}$. We need only apply the formula from Carmichael $[2$, p. 328] which in our notation is:

$$
N_{h}^{m}\left(p^{s}\right)=\frac{\left(p^{(m+1) s}-1\right)\left(p^{m s}-1\right) \cdots\left(p^{(m-h+1) s}-1\right)}{\left(p^{(h+1) s}-1\right)\left(p^{h s}-1\right) \cdots\left(p^{s}-1\right)} .
$$


This is the number of $h$-dimensional subspaces $\operatorname{PG}\left(h, p^{s}\right)$ in a $\mathrm{PG}\left(m, p^{s}\right)$. To abbreviate the notation let $n=p^{s}$ :

$$
\begin{aligned}
& N_{h}^{m}(n)=\frac{\left(n^{m+1}-1\right)\left(n^{m}-1\right) \cdots\left(n^{m-h+1}-1\right)}{\left(n^{h+1}-1\right)\left(n^{h}-1\right) \cdots(n-1)}, \\
& N_{m}^{m}(n)=1=N_{-1}^{m}(n) .
\end{aligned}
$$

In terms of rank this is the number of elements of rank $H=h+1$ in the lattice $L=\operatorname{PG}\left(m, p^{s}\right)$, which itself is of rank $M=m+1$ :

$$
\begin{aligned}
& T_{H}^{M}(n)=\frac{\left(n^{M}-1\right)\left(n^{M-1}-1\right) \cdots\left(n^{M-H+1}-1\right)}{\left(n^{H}-1\right)\left(n^{H-1}-1\right) \cdots(n-1)}, \\
& T_{M}^{M}(n)=1=T_{0}^{M}(n) .
\end{aligned}
$$

By reducing the fraction to its lowest terms we obtain the symmetry property enjoyed also by the binomial coefficients:

$$
T_{M-H}^{M}(n)=T_{H}^{M}(n) \quad(H=0,1, \cdots, M) .
$$

This states that the number of elements rank $M-H$ is equal to the number of elements of rank $I I$. The fact that $\operatorname{PG}\left(m, p^{s}\right)$ possesses a dual automorphism of course also demands this. In terms of dimension:

$$
N_{m-(h+1)}^{m}(n)=N_{h}^{m}(n) \quad(h=-1,0,1, \cdots, m) .
$$

We desire to interpret this as follows: The number of $[m-(h+1)]$-dimensional spaces (containing the null space) is equal to the number of $h$-dimensional spaces (contained in the entire space $\mathrm{PG}\left(m, p^{s}\right)$ ).

The classification of elements of $L=\operatorname{PG}\left(m, p^{s}\right)$ as $x$ 's and as $y$ 's now follows:

$$
\{u\} \quad V(1,0, \cdots, 0) \quad W\left(1, T_{1}^{M}, T_{2}^{M}, \cdots, T_{M-1}^{M}, 1\right)
$$

$\left\{\begin{array}{l}\text { Set of elements of } \\ \text { rank } M-H \\ (H=0,1, \cdots, M)\end{array}\right\}$

$$
\begin{aligned}
& V\left(1, T_{1}^{H}, T_{2}^{H}, \cdots, T_{I I}^{I I}=1,0, \cdots, 0\right) \\
& \quad W\left(1, T_{1}^{M-H}, T_{2}^{M-I I}, \cdots, T_{M-H}^{M-I I}=1,0, \cdots, 0\right)
\end{aligned}
$$

$\left\{\begin{array}{l}\text { Sets of elements of } \\ \text { rank } H \\ (H=0,1, \cdots, M)\end{array}\right\}$

$$
\begin{array}{r}
V\left(1, T_{1}^{M-H I}, T_{2}^{M-H}, \cdots, T_{M-H}^{M-H}=1,0, \cdots, 0\right) \\
W\left(1, T_{1}^{H}, T_{2}^{H}, \cdots, T_{H}^{H}=1,0, \cdots, 0\right) \\
V\left(1, T_{1}^{M}, T_{2}^{M}, \cdots, T_{M-1}^{M}, 1\right) \quad W(1,0, \cdots, 0)
\end{array}
$$$$
\{z\}
$$

This completes the proof and discussion of Lemma 3.5. 
Lenma 3.6. Theorem 2 is true for an arbitrary finite complemented modular lattice $L_{C}$.

Proof. Every finite complemented modular lattice is uniquely the direct product of finite projective geometries [1, p. 120, Theorem 6] and a Boolean algebra $\beta^{n}=B \times \cdots \times B$ ( $n$ factors) $n \geqq 0$. Veblen and Bussey [4] proved that every finite projective geometry of dimension $m>2$ is a $\mathrm{PG}\left(m, p^{s}\right)$, the lattice of subspaces of the $(m+1)$-dimensional vector space of $(m+1)$-dimensional vectors with homogeneous coordinates from a finite field $\mathrm{GF}\left(p^{s}\right)$. Hence $L_{C}$ is the direct product of $B^{\prime}$ s, regular projective lines $\operatorname{PG}\left(1, p^{s}\right)$ and exceptional projective lines $\operatorname{PG}(1, n)$, nonDesarguesian planes $\operatorname{PG}(2, n)$, and projective geometries $\operatorname{PG}\left(m, p^{s}\right)(m \geqq 2)$ wherein Desargue's Theorem is always valid $[2$, p. 337]. We have proved the validity of Theorem 2 for each of these types in Lemmas 3.2-3.5 respectively. Hence $L_{C}=L_{1} \times \cdots \times L_{t}$ where each $L_{i}$ is a projective geometry of one of the types above and for which Theorem 2 is valid. Let $\left[a_{1}, \cdots, a_{t}\right]$ and $\left[b_{1}, \cdots, b_{t}\right]$ be the standard component representation for elements $a$ and $b$ of $L_{C}: a_{i}, b_{i} \in L_{i},(i=1, \cdots, t)$. Then $a \supseteq b$ if and only if $a_{i} \supseteq b_{i}$ in $L_{i},(i=1, \cdots, t)$; and $a \cup b$ and $a \cap b$ are obtained by taking joins and meets component-wise. Every quotient sublattice $a / b$ of $L_{C}$ is complemented, and indeed $a / b$ is a direct product:

$$
\frac{a}{b}=\frac{a_{1}}{b_{1}} \times \cdots \times \frac{a_{t}}{b_{t}}
$$

where $a_{i} / b_{i}$ is a projective subgeometry of $L_{i}$ of same or lower dimension than $L_{i},(i=1, \cdots, t)$. For $i=1, \cdots, t$ let $Q_{i 0}, Q_{i 1}, \cdots, Q_{i M_{i}}$ be the set of all complete sets of projective (necessarily complemented) quotient sublattices of $L_{i}$ of length $M_{i}$, where $Q_{i H_{i}}$ is the set of all subspaces of length $H_{i}$, $0 \leqq H_{2} \leqq M_{i}$. When $a_{i} / b_{i}$ ranges over a fixed $Q_{i H_{i}},(i=1, \cdots, t)$, then $a / b$ ranges over a complete $Q_{H_{1}}, \ldots, H_{t}$ of $L_{C}$ itself. For each distinct selection of the values of $H_{1}, \cdots, H_{t}$ one obtains a distinct complete set $Q_{H_{1}}, \cdots, H_{t}$, and no other complete sets of projective (complemented) quotient sublattices than these exist in $L_{C}$. More detailed verification of these remarks is routine.

Corresponding $V$ and $W$ sets for $L_{C}$ may now be described. The $W$ set is the set of complements of the $V$ set. Starting with $x=\left[x_{1}, \cdots, x_{t}\right] \in V$, for each $i$ let $x_{i}$ range independently over the set $V_{i}$ of $L_{i}$ to which it belongs, namely the elements of same rank $H_{i}$ (of $x_{i}$ ) in $L_{i}$. Then the set $W$ of $y$ $=\left[y_{1}, \cdots, y_{t}\right]$ corresponding to $V$ is obtained by letting, for each $i$, the component $y_{i}$ range independently over the set $W_{i}$ corresponding to $V_{i}$ in $L_{i}$, namely the elements of $L_{i}$ of complementary rank $M_{i}-H_{i}$ in $L_{i}$. The order of $V$ is the product of the orders of the $V_{i}$, formulas for which are given in Lemmas 3.2 to 3.5. The order of $W$ is the product of the orders of the $W_{i}$. Since the orders of corresponding $V_{i}$ and $W_{i}$ are equal by Lemmas 3.2 to 3.5, this yields equality of the orders of $V$ and $W$. The proof of Lemma 3.6 is now complete. 
Again we emphasize that a $W$ set is the set of complements of the corresponding $V$ set.

We turn next to the proof of Theorem 2 for an arbitrary finite modular lattice.

\section{The general finite modular case.}

The proof of Theorem 2 for an arbitrary finite modular lattice $L$ we now undertake in a sequence of lemmas ending as one would expect by an induction. Techniques employed by Dilworth [3] are applied. In the following lemmas $a^{*}$ and $a_{*}$ are as defined in the statement of Theorem 2.

Lemma 4.1. In a finite modular lattice $a \cap x^{*}=x$ implies $a=x$, and dually $a \cup x_{*}=x$ implies $a=x$.

Assume $a \supset x$ (proper inclusion). Then, by the descending chain condition, there exists $v$ such that $a \supseteq v>x(v$ covers $x)$. Since $x^{*}$ is the join of all elements covering $x, x^{*} \supseteq v$. This yields $x=a \cap x^{*} \supseteq v>x$, a contradiction. Hence $a=x$. The second part follows dually.

Definition 4.1. We define a Weisner-Möbius (dual) function $\mu(x)$ inductively downward from the unit element $u \in L$ as follows:

$$
\begin{aligned}
& \mu(u)=1, \\
& \mu(a)=-\sum_{x \supset a} \mu(x) \quad \text { for } a \subset u .
\end{aligned}
$$

We can combine these two formulas:

$$
\sum_{x \supseteq a} \mu(x)=\delta_{a}^{u}
$$

where $\delta_{a}^{u}$ is the Kronecker $\delta: \delta_{a}^{u}=1$ or 0 according as $a=u$ or $a \neq u$.

LeMma 4.2 .

$$
\sum_{\boldsymbol{x} \cap b=a} \mu(x)=\mu(a) \cdot \delta_{b}^{u} .
$$

Proof. We make an induction downward on $a$. When $a=u, u=b=x=a$ and we obtain

$$
\begin{aligned}
\sum_{x=u} \mu(x) & =\mu(u) \\
& =1=\mu(a) \cdot \delta_{u}^{u} .
\end{aligned}
$$

Assume the lemma is true for all $c \supset a$. For $b=u$ we have

$$
\sum_{x \cap b=a} \mu(x)=\sum_{x=a} \mu(x)=\mu(a) \cdot \delta_{u}^{u} 1
$$

as desired. For $a \subseteq b \subset u$ and dummy variables $x$ and $c$ 


$$
\begin{aligned}
\sum_{\mathbf{x} \cap b=a} \mu(x) & =\sum_{\mathbf{x} \cap b=a} \mu(x)+\sum_{\mathbf{c} \supset a}[0] \\
& =\sum_{\mathbf{x} \cap b=a} \mu(x)+\sum_{b \supseteq c \supset a}\left[\sum_{\mathbf{x} \cap b=c} \mu(x)\right] \\
& =\sum_{b \supseteq c \supseteq a} \sum_{x \in b=c} \mu(x) \\
& =\sum_{x \supseteq a} \mu(x)=\delta_{a}^{u} \\
& =0=\mu(a) \cdot \delta_{b}^{u}, \text { since } \delta_{b}^{u}=0 .
\end{aligned}
$$

In the fourth step we note that the dual ideal of all $x \supseteq a$, with $b \supseteq a$, can be partitioned into disjoint subsets of $x$ 's satisfying $x \cap b=c$, where $c$ ranges over the quotient lattice $b / a$. The induction is thus complete.

It is also easy to show that $\mu(x)=0$ for all $x \nsupseteq u_{*}$ by a downward induction in the partially ordered set $L-\left(u / u_{*}\right)$. However, we shall not need this property.

Definition 4.2. Let $J(L)=\{y \in L \mid y$ has property $j$ in $L\}$. Let $J(a)$ $=\{y \subseteq a \mid y$ has property $j$ in $L\}$ so that $J(a)=J(L) \cap(a / z)$. Let $T(b)$ $=\left\{y \in J(L) \mid y \cup u_{*}=b\right\}$.

Notation. $N[S]$ is the order of the set $S$.

Lemma 4.3. For $a \supseteq u_{*}$, the union of scts

$$
J(a)=\underset{u * \subseteq b \subseteq a}{\bigcup} T(b)
$$

is a partitioning of $J(a)$ into disjoint subsets. And thus

$$
N[J(a)]=\sum_{u * \subseteq b \subseteq a} N[T(b)] .
$$

For $u_{*} \subseteq b \subseteq a, y \in T(b)$ implies $y$ has property $j$ in $L$ and $y \subseteq y \cup u_{*}=b \subseteq a$, so that $y \in J(a)$. Conversely $y \in J(a)$ implies that $y$ belongs to a unique $T(b)$ determined by $b=y \cup u_{*}$.

LEMMA 4.4.

$$
N[T(u)]=\begin{array}{ll}
1 & \text { if } u \in J(L), \\
0 & \text { otherwise. }
\end{array}
$$

By Lemma $4.1 y \cup u_{*}=u$ requires $y=u$. Hence by Definition 4.2 $T(u)$ consists of $u$ only when $u$ has property $j$ and is otherwise empty.

Notation. Henceforth let $L_{*}$ be the dual ideal $u / u_{*}$. Whence $L_{*}$ is the complemented modular sublattice at the top of $L$ generated by its dual points. 
Lemma 4.5.

$$
\sum_{a \in L_{*}} \mu(a) N[J(a)]=\begin{array}{ll}
1 & \text { if } u \in J(L), \\
0 & \text { otherwise. }
\end{array}
$$

By Lemma 4.3

$$
\begin{aligned}
\sum_{a \in L_{*}} \mu(a) N[J(a)] & =\sum_{a \supset u_{*}} \mu(a) \sum_{a \supseteq b \supseteq u_{*}} N[T(b)] \\
& =\sum_{a \supset u_{*}} N[T(b)] \sum_{a \supset b} \mu(a) \\
& =\sum_{b \supseteq u_{*}} N[T(b)] \cdot \delta_{b}^{u} \\
& =N[T(u)] \\
& =1 \quad \text { if } u \in J(L), \\
& 0 \text { _otherwise. }
\end{aligned}
$$

Definition 4.1

Lemma 4.4

In our further development we shall be concerned in two ways with the ideals $a / z$ where $a$ ranges over $L_{*}$ : first, as lattices in their own right with their own complete sets of projective complemented quotients; and secondly, as sublattices of $L$ with their complemented quotient sublattices found among the $Q_{i}$ of $L$. Projectivity in $a / z$ of two complemented quotients of $a / z$ trivially implies their projectivity in $L$, but projectivity in $L$ does not necessitate their projectivity in $a / z$.

Definition 4.3. For the ideal $a / z$ as a lattice in its own right, let $Q_{a 0}$, $Q_{a 1}, \cdots, Q_{a r(a)}$ be its complete sets of projective complemented quotients, and let $V\left(h_{0}, h_{1}, \cdots, h_{r(a)} ; a / z\right)$ and $W\left(h_{0}, h_{1}, \cdots, h_{r(a)} ; a / z\right)$ represent its $V$ and $W$ sets.

Definition 4.4. Let $V\left(k_{0}, k_{1}, \cdots, k_{r} ; a\right)$ be the set of all $x \subseteq a$ for which there are precisely $k_{i}$ quotients belonging to $Q_{i}(i=0,1, \cdots, r)$ that are minimal in the complemented quotient sublattice $x_{a}^{*} / x$ of $a / z$ and therefore have denominator $x$, where we have let $x_{a}^{*} \equiv x^{*} \cap a$. Let $W\left(k_{0}, k_{1}, \cdots, k_{r} ; a\right)$ be the set of all $y \subseteq a$ for which there are precisely $k_{i}$ quotients belonging to $Q_{i}(i=0,1, \cdots, r)$ that are maximal in $y / y_{*}$ of $a / z$ and therefore have numerator $y$.

Notation. Hereafter we shall abbreviate the $(r+1)$-tuple $\left(k_{0}, k_{1}, \cdots, k_{r}\right)$ by $(K)$ whenever no ambiguity arises thereby.

REMARKs. The quotient $x_{a}^{*} / x$ is the complemented quotient sublattice of $a / z$ generated above $x, x \subseteq a$, by the elements in $a / z$ that cover $x$. It is the intersection of $x^{*} / x$ and $a / z$. On the other hand for $y \subseteq a$ we have $y_{*} \subseteq y \subseteq a$. Hence $\left(y_{a}\right)_{*} \equiv y_{*} \cap a=y_{*}$ and $y /\left(y_{a}\right)_{*}=y / y_{*}$ so that $W(K ; a)=W(K) \cap(a / z)$. No property similar to the latter holds for the $V$ 's, since for some $x \subseteq a$ it 
may happen that $x^{*} \Phi a$ and $x^{*} / x$ is not a quotient of $a / z$. I.e., for $x \subseteq a$, $x \in V(K ; a)$ and $x \in V(K)$ are not equivalent.

LEMMA 4.6.

$$
\sum_{a \in L_{*}} \mu(a) N[W(K ; a)]=\begin{array}{ll}
1 & \text { if } u \in W(K), \\
0 & \text { otherwise. }
\end{array}
$$

Apply Lemma 4.5 with property $j: y \in W(K)$ and recall that $y \in W(K)$ and $y \subseteq a$ imply $y \in W(K ; a)$.

Lemma 4.7. Validity of Theorem 2 in the ideal quolient lattice $a / z$ :

$$
N\left[V\left(h_{0}, \cdots, h_{r(a)} ; a / z\right)\right]=N\left[W\left(h_{0}, \cdots, h_{r(a)} ; a / z\right)\right]
$$

for all sets of values of the $h_{i}$ implies:

$$
N\left[V\left(k_{0}, \cdots, k_{r} ; a\right)\right]=N\left[W\left(k_{0}, \cdots, k_{r} ; a\right)\right]
$$

for all choices of the $k_{i}$.

Proof. For an arbitrary fixed $a \in L$ and each $i,(i=0,1, \cdots, r)$, the projective complemented quotients in the complete set $Q_{i}$ of $L$ found in $a / z$ are not necessarily all projective in $a / z$, but the set of all such are further partitioned into disjoint complete sets of projective quotients in $a / z: Q_{i 1}, \cdots$, $Q_{i a(i)}$. Now $Q_{i j}=Q_{m n}$ if and only if $i=m$ and $j=n$, since projectivity in $a / z$ implies projectivity in $L$. Every element of $a / z$ is an $x$ of a unique $V$ set and is also a $y$ of a unique $W$ set of $a / z$. Employing the above alternative double subscript notation with respect to the projective set $Q_{i j}$, let $V\left(h_{01}, \cdots, h_{0 a(0)}\right.$, $\left.\cdots, h_{r 1}, \cdots, h_{r a(r)} ; a / z\right)$ and $W\left(h_{01}, \cdots, h_{0 a(0)}, \cdots, h_{r 1}, \cdots, h_{r a(r)}\right.$; $a / z)$ be arbitrary corresponding $V$ and $W$ sets in $a / z$. They are of the same order by, hypothesis. When these same corresponding $V$ and $W$ sets are looked at from the point of view of projectivity in $L$ rather than in $a / z$, $Q_{i 1}, \cdots, Q_{i a(i)}$ are identified as belonging to $Q_{i}$ and we get for each $x \in V$, $x$ is the denominator of $k_{i}=h_{i 1}+\cdots+h_{i a(i)}$ quotients of $Q_{i}$; and for each $y \in W, y$ is the numerator of $k_{i}=h_{i 1}+\cdots+h_{i a(i)}$ quotients of $Q_{i}$. Since the $V$ and $W$ sets are precisely the same sets as before except for notation, they remain of equal order. For each of the sets $Q_{j}$ of $L$ that has no quotient in $a / z$ the corresponding $k_{j}$ is zero.

Definition 4.5. Let $S(K ; x)=\left\{a \in L_{*} \mid x \in V(K ; a)\right\}$.

REMARK. Recall $x$ is not necessarily an element of $V(K)$.

Definition 4.6. For a fixed $x \notin L_{*}$ let $R_{x}$ be the binary relation defined on the elements of $L_{*}$ by

$$
a R_{x} b \text { if and only if } a \cap\left(x^{*} \cup u_{*}\right)=b \cap\left(x^{*} \cup u_{*}\right) .
$$

Lemma 4.8. For $x \notin L_{*}, R_{x}$ is an equivalence relation partitioning $L_{*}$ into disjoint equivalence classes $A_{x}, B_{x}, \cdots$, such that for each class $A_{x}$ 


$$
\sum_{a \in A_{x}} \mu(a)=0 .
$$

That $R_{x}$ is an equivalence relation is obvious. Let $a_{0}$ be a fixed element of the equivalence class $A_{x}$. Applying Lemma 4.2 with $a, x^{*} \cup u_{*}, a_{0} \cap\left(x^{*} \cup u_{*}\right)$ taking the roles of $x, b, a$ respectively we obtain:

$$
\begin{aligned}
\sum_{a \in A_{x}} \mu(a) & =\sum_{a \cap\left(x_{*} \cup u_{*}\right)=a_{0} \cap\left(x_{*} \cup_{\left.u_{*}\right)}\right.} \mu(a) \\
& =\mu\left(a_{0} \cap\left(x^{*} \cup u_{*}\right)\right) \cdot \delta_{x_{*} \cup_{u_{*}}}^{u}
\end{aligned}
$$

But $x^{*} \cup u_{*} \neq u$. Otherwise $x^{*}=u$ by Lemma 4.1 ; and since $x$ is the meet of the dual points of the complemented modular sublattice $x^{*} / x[1$, Dual to Corollary to Theorem 1, p. 114];

$$
x=\bigcap_{x \subseteq q<x^{*}} q \supseteq \bigcap_{q<x^{*}} q=\left(x^{*}\right)_{*}=u_{*}
$$

contrary to hypothesis. Thus the Kronecker delta has value zero, making the proof complete.

Lemma 4.9. For $x \notin L_{*}, S(K ; x)$ is the set union of equivalence classes, determined by $R_{x}$, which it contains.

The lemma holds vacuously for empty $S(K ; x)$. Next let $S(K ; x)$ have an element $a$ in common with an equivalence class $A_{x}$ and let $b$ be any other element of $A_{x}$. We shall show $b \in S(K ; x)$. By the modular law

$$
\begin{array}{rlr}
x_{a}^{*} \equiv a \cap x^{*} & =a \cap\left(x^{*} \cup u_{*}\right) \cap x^{*} & \\
& =\left[\left(a \cap x^{*}\right) \cup u_{*}\right] \cap x^{*} & \text { since } a \supseteq u_{*} \\
& =\left[\left(b \cap x^{*}\right) \cup u_{*}\right] \cap x^{*} & \\
& =b \cap\left(x^{*} \cup u_{*}\right) \cap x^{*} & \text { since } b \supseteq u_{*} \\
& =b \cap x^{*} \equiv x_{b}^{*} .
\end{array}
$$

Now $a \in S(K ; x)$ implies $x \in V(K ; a)$, which states that $x$ is the denominator of $k_{i}$ quotients of $Q_{i}(i=0,1, \cdots, r)$ in $x_{a}^{*} / x=x_{b}^{*} / x$. Hence $x \in V(K ; b)$. Thus $b \in S(K ; x)$. Since $b$ was arbitrarily chosen in $A_{x}, A_{x} \subseteq S(K ; x)$. It follows that $S(K ; x)$ is the set union of the equivalence classes which it contains, and the proof is complete.

Lemma 4.10. For $x \in L_{*}, \quad \sum_{a \in S(K ; x)} \mu(a)=0$.

By the preceding Lemmas 4.9 and 4.8

$$
\begin{aligned}
\sum_{a \in S(K ; x)} \mu(a) & =\sum_{A_{x} \subseteq S(K ; x)} \sum_{a \in A_{x}} \mu(a) \\
& =\sum_{A_{x} \subseteq S(K ; x)} 0=0 .
\end{aligned}
$$

For the following definition we note that Definition 4.5 essentially states that $a \in S(K ; x)$ if and only if $x \in V(K ; a)$. 
DEFINITION 4.7. Let

LEMMA 4.11.

$$
\phi(K ; a, x)=\begin{aligned}
& 1 \quad \text { if }\left\{\begin{array}{l}
a \in S(K ; x) \\
x \in V(K ; a)
\end{array}\right\} \\
& 0 \text { otherwise. }
\end{aligned}
$$

$$
\sum_{a \in L_{*}} \mu(a) N[V(K ; a)]=\begin{array}{ll}
1 & \text { if } u \in W(K) \\
0 & \text { otherwise. }
\end{array}
$$

We cannot apply Lemma 4.5 to obtain this result as we did to obtain Lemma 4.6, since $x \in V(K)$ and $x \subseteq a$ do not imply $x \in V(K ; a)$.

$$
\begin{aligned}
\sum_{a \in L_{*}} \mu(a) N[V(K ; a)] & =\sum_{a \in L_{*}} \mu(a) \sum_{x \in L} \phi(K ; a, x) \\
& =\sum_{x \in L} \sum_{a \in L_{*}} \mu(a) \phi(K ; a, x) \\
& =\sum_{x \in L} \sum_{a \in S(K ; x)} \mu(a) \\
& =\sum_{x \in L_{*}} \sum_{a \in S(K ; x)} \mu(a) \quad \text { by Lemma 4.10 } \\
& =\sum_{x \in L_{*}} \sum_{a \in L_{*}} \mu(a) \phi(K ; a, x) \\
& =\sum_{a \in L_{*}} \mu(a) \sum_{x \in L_{*}} \phi(K ; a, x) \\
& =\sum_{a \in L_{*}} \mu(a) N\left[V_{*}(K ; a)\right]
\end{aligned}
$$

where $V_{*}(K ; a)=V(K ; a) \cap L_{*}$. Since $L_{*}$ is complemented, for every $x \in L_{*}$ the quotient sublattice $u / x$ of $L_{*}$ is also complemented and $u=x^{*}$, the join of the elements covering $x[1$, Corollary to Theorem 1, p. 114]. Hence $x_{a}^{*} \equiv x^{*} \cap a=u \cap a=a$. Thus $V_{*}(K ; a)$ can be described as the set of $x \in a / u_{*}$ for which there are precisely $k_{i}$ quotients belonging to $Q_{i}(i=0,1, \cdots, r)$ that are minimal in the complemented quotient $a / x=x_{a}^{*} / x$ of $a / u_{*}$, therefore having denominator $x$. Let $W_{*}(K ; a)$ be the set of $y$ in $a / u_{*}$ for which there are precisely $k_{i}$ quotients belonging to $Q_{i}(i=0,1, \cdots, r)$ that are maximal in the complemented quotient $y / u_{*}$ of $a / u_{*}$, therefore having numerator $y$.

We shall next show that $N\left[V_{*}(K ; a)\right]=N\left[W_{*}(K ; a)\right]$.

Definition $4.3_{*}$. For the ideal $a / u_{*}$ as a lattice in its own right let $Q_{* a 0}, Q_{* a 1}, \cdots, Q_{* a r_{*}(a)}$ be its complete sets of (necessarily complemented) projective quotients and let

$$
V_{*}\left(h_{0}, h_{1}, \cdots, h_{r_{*}(a)} ; a / u_{*}\right) \text { and } W_{*}\left(h_{0}, h_{1}, \cdots, h_{r_{*}(a)} ; a / u_{*}\right)
$$

represent its $V$ and $W$ sets.

Lemma 3.6 asserts that Theorem 2 is valid in the complemented lattice $a / u_{*}:$

$$
N\left[V_{*}\left(h_{0}, \cdots, h_{r_{*}(a)} ; a / u_{*}\right)\right]=N\left[W_{*}\left(h_{0}, \cdots, h_{r_{*}(a)} ; a / u_{*}\right)\right]
$$


We proceed now with the type of argument found in the proof of Lemma 4.7. For a fixed $a \in L_{*}$ and each $i(i=0,1, \cdots, r)$ those complemented quotients of a complete set $Q_{i}$ of $L$ found in $a / u_{*}$ are not necessarily projective in $a / u_{*}$, but the set of all such are further partitioned into disjoint complete sets of projective (complemented) quotients in $a / u_{*}: Q_{* i 1}, \cdots, Q_{* i a(i)}$. We complete the argument of Lemma 4.7 applied now to $a / u_{*}$ rather than to $a / z$. The above equality of orders of corresponding $V$ and $W$ sets of $a / u_{*}$, from the point of view of projectivity in $L$ instead of in $a / u_{*}$, now reduces to

$$
N\left[V_{*}(K ; a)\right]=N\left[W_{*}(K ; a)\right] .
$$

Combining this with our initial equality we obtain

$$
\sum_{a \in L_{*}} \mu(a) N[V(K ; a)]=\sum_{a \in L_{*}} \mu(a) N\left[W_{*}(K ; a)\right] .
$$

Let $W_{*}(K)=W_{*}(K ; u)$, which is the set of $y \in L_{*}$ for which there are precisely $k_{i}$ quotients belonging to $Q_{i}(i=0,1, \cdots, r)$ that are maximal in $y / u_{*}$ of $L$, therefore having numerator $y$ (and denominator containing $u_{*}$ ). Clearly again here as in the unstarred case $W_{*}(K ; a)=W_{*}(K) \cap\left(a / u_{*}\right)$. Applying Lemma 4.5 to $L_{*}$ with property $j: y \in W_{*}(K)$, we obtain

$$
\sum_{a \in L_{*}} \mu(a) N\left[W_{*}(K ; a)\right]=\begin{array}{ll}
1 & \text { if } u \in W_{*}(K), \\
0 & \text { otherwise. }
\end{array}
$$

But by the definitions of $W_{*}(K)$ and $W(K), u \in W_{*}(K)$ if and only if $u$ is the numerator of precisely $k_{i}$ quotients of $Q_{i}(i=0,1, \cdots, r)$ falling in $u / u_{*}=L_{*}$ if and only if $u \in W(K)$. Combining our results

$$
\sum_{a \in L_{*}} \mu(a) N\left[W_{*}(K ; a)\right]=\begin{array}{ll}
1 & \text { if } u \in W(K), \\
0 & \text { otherwise. }
\end{array}
$$

This completes the proof of Lemma 4.11.

5. Proof of the principal theorem. We now prove Theorem 2 by induction. It is trivially true for the lattices of length 0 and 1 as already stated in Lemmas 3.1 and 3.2. Assume that the theorem is true for all proper quotient lattices of a finite modular lattice $L$. We get

$$
\begin{aligned}
0= & \sum_{a \in L_{*}} \mu(a)\{N[V(K ; a)]-N[W(K ; a)]\} \text { by Lemmas 4.6, 4.11 } \\
= & \mu(u)\{N[V(K ; u)]-N[W(K ; u)]\} \\
& +\sum_{u * \subseteq a \subset_{u}} \mu(a)\{N[V(K ; a)]-N[W(K ; a)]\} \\
= & 1 \cdot\{N[V(K)]-N[W(K)]\} \quad \text { by Definition 4.1, Lemma 4.7. } \\
& +\sum_{u * \subseteq a \subset_{u}} \mu(a) \cdot\{0\}
\end{aligned}
$$


Hence we conclude

$$
N[V(K)]=N[W(K)]
$$

This completes the induction.

6. Special cases. Having shown that corresponding $V$ and $W$ sets have the same order we next show the relationship between the $x \in V$ and $y \in W$ in Theorems 6.1 and 6.2. First we prove some preliminary lemmas.

Lemma 6.1. A complemented quotient sublattice of a finite modular lattice $L$ is a maximal quotient of the complete set $Q_{m}$ of projective quotients to which it belongs if and only if it is of the form $x^{*} / x$. And dually it is minimal in $Q_{m}$ if and only if it is of the form $y / y_{*}$.

Proof. Let $v / x$ be maximal in $Q_{m}$. For every $p_{i}$ covering $x, p_{i} \subseteq v$; otherwise $v / x$ is a lower transpose of $p_{i} \cup v / p_{i}$, a contradiction. Since $v / x$ is complemented, $v=x^{*}$, the join of the $p_{i}[1$, Corollary 1 to Theorem 1, p. 114]. Conversely we show $x^{*} / x$ has no proper upper transpose. If $a / b$ is an upper transpose of $x^{*} / x$, then $b \cap x^{*}=x$, and by Lemma $4.1 b=x, a=b \cup x^{*}=x^{*}$.

Lemma 6.2. The set of denominators $x_{1}, x_{2}, \cdots$, of all maximal quotients $x_{1}^{*} / x_{1}, x_{2}^{*} / x_{2}, \cdots$ of a complete set $Q_{m}$ of projective complemented quotients of a finite modular lattice $L$ constitute a complete set $V\left[k_{0}, k_{1}, \cdots, k_{r}\right]$ of Theorem 2 ; and dually, the numerators $y_{1}, y_{2}, \cdots$ of the minimal quotients $y_{1} / y_{1 *}$, $y_{2} / y_{2 *}, \cdots$ of $Q$ constitute a complete set $W\left[k_{0}^{\prime}, k_{1}^{\prime}, \cdots, k_{r}^{\prime}\right]$.

Proof. The only complemented quotients of form $a / x$ in $L$ are in $x^{*} / x^{*}$ For $a$ is a join of elements covering $x$ [1, Corollary to Theorem 1, p. 114] which are, by definition of $x^{*}$, contained in $x^{*}$, making $a \subseteq x^{*}$. We next apply to an arbitrary pair $x_{1}^{*} / x_{1}, x_{2}^{*} / x_{2}$ of maximal quotients of $Q_{m}$ Theorem 6 of Birkhoff $[1$, p. 73] and its extension to projective quotients. This theorem states that a natural isomorphism between transposed quotients (say $a \cup b / a$ and $b / a \cap b$ given by the 1-1 correspondence $x \leftrightarrow a \cup x$, where $x \in(b / a \cap b)$ and $(a \cup x) \in(a \cup b / a))$ carries subquotients into transposed subquotients. The 1-1 correspondence between $x_{1}^{*} / x_{1}$ and $x_{2}^{*} / x_{2}$ determined by the sequence of natural isomorphisms between successive transposed quotients of a chain of such between $x_{1}^{*} / x_{1}$ and $x_{2}^{*} / x_{2}$ yields an isomorphism of these quotients that carries each ideal $a_{1} / x_{1}$ of $x_{1}^{*} / x_{1}$ into a unique projective ideal $a_{2} / x_{2}$ of $x_{2}^{*} / x_{2}$. I.e., the ideals of $x_{1}^{*} / x_{1}$ and of $x_{2}^{*} / x_{2}$ are pairwise projective. In view of this and the first remark of the proof, $x_{1}$ and $x_{2}$ belong to the same $V\left(k_{0}, \cdots, k_{r}\right)$. We note that $k_{m}=1$.

Conversely we shall show that any $x$ belonging to this same $V\left(k_{0}, \cdots, k_{r}\right)$ will determine a maximal quotient $x^{*} / x$ of $Q_{m}$. For such an $x, x^{*} / x$ possesses precisely $k_{m}=1$ ideals $v / x$ belonging to $Q_{m}$. Assume $x^{*} \supset v$. Then $x^{*} / x$ is a member of a set $Q_{n}$ of projective complemented quotients of greater length than $v / x, x_{1}^{*} / x_{1}$, and the other members of $Q_{m}$; and $k_{n} \geqq 1$. But regarding 
$x, x_{1} \in V\left(k_{0}, \cdots, k_{m}=1, \cdots, k_{n} \geqq 1, \cdots, k_{r}\right)$, we get $x_{1}^{*} / x_{1}$ possessing $k_{n} \geqq 1$ ideals in $Q_{n}$ of length greater than $x_{1}^{*} / x_{1}$ itself, a contradiction. Hence the assumption is false and $x^{*} / x=v / x$ belongs to $Q_{m}$. This completes the proof of Lemma 6.2.

Lemma 6.3. The sets $V\left(k_{0}, \cdots, k_{r}\right)$ and $W\left(k_{0}^{\prime}, \cdots, k_{r}^{\prime}\right)$ determined as in Lemma 6.2 by the same set $Q_{m}$ of complemented projective quotients are corresponding $V$ and $W$ sets: $k_{i}=k_{i}^{\prime}(i=0,1, \cdots, r)$.

Proof. Let $x^{*} / x$ and $y / y_{*}$ be arbitrary maximal and minimal quotients respectively of $Q_{m}$. As in the proof of Lemma 6.2, the 1-1 correspondence between $x^{*} / x$ and $y / y_{*}$ determined by the sequence of natural isomorphisms between successive transposed quotients of a chain of such between them yields an isomorphism that pairs off the ideals of $x^{*} / x$ and the ideals of $y / y_{*}$ projectively. We next show that the ideals of $y / y_{*}$ may be paired off in a 1-1 manner projectively with its dual ideals $y / b$. For we can apply Lemma 3.6 to $y / y_{*}$, since it is complemented, noting as we did at the conclusion of its proof that each $W$ set of $y / y_{*}$ is the set of complements in $y / y_{*}$ of the elements of the corresponding $V$ set. But complements $c$ and $c^{\prime}$ of $y / y_{*}$ yield a transposed ideal $c / y_{*}$ and dual ideal $y / c^{\prime}$. Thus, equality of the orders of the corresponding $V$ and $W$ sets means that ideals and dual ideals of $y / y_{*}$ can be paired off in projective pairs. We thus obtain finally that the ideals of $x^{*} / x$ may be paired off projectively in a 1-1 manner with the dual ideals of $y / y_{*}$. As shown in the proof of Lemma 6.2 the only complemented quotients $a / x$ of $L$ are in $x^{*} / x$ and dually the only complemented quotients $y / b$ are in $y / y_{*}$. Hence $k_{i}=k_{i}^{\prime}(i=0,1, \cdots, r)$ and $x$ and $y$ belong to corresponding $V$ and $W$ sets. This completes our proof of Lemma 6.3.

Combining Lemmas 6.2 and 6.3 we get

THEOREM 6.1. Associated with each complete set $Q$ of projective complemented quotients of a finite modular lattice is a unique pair of corresponding $V$ and $W$ sets in which the $x \in V$ are the denominators of the maximal quotients $x^{*} / x$ and the $y \in W$ are the numerators of the minimal quotients $y / y_{*}$ of $Q$.

THEOREM 6.2. The number of maximal quotients of a complete set $Q$ of projective complemented quotients of a finite modular lattice is equal to the number of minimal quotients.

Proof. Combine Theorem 2 and Theorem 6.1.

Definition 6.1. In accordance with Theorem 1.1, with each complete set $Q$ of prime quotients of a finite modular lattice are associated meet irreducibles which are the denominators of the maximal prime quotients of $Q$ and join irreducibles which are the numerators of the minimal quotients of $Q$.

COROLlaRy 6.2.1. The number of meet irreducibles associated with each complete set $Q$ of prime quotients of a finite modular lattice is equal to the number of join irreducibles associated with $Q$. 
Summing over all sets of prime quotients of $L$ we obtain the simpler of Dilworth's two principal results in [3]:

COROLlary 6.2.2. The number of meet irreducibles of a finite modular lattice is equal to the number of join irreducibles.

THEOREM 6.3. Let $Q_{1}^{\prime}, Q_{2}^{\prime}, \cdots, Q_{s}^{\prime}$ be an arbitrary subset of the set of all sets of projective complemented quotients $Q_{0}, Q_{1}, \cdots, Q_{r}$ of a finite modular lattice $L$, and let $Q_{1}^{\prime \prime}, Q_{2}^{\prime \prime}, \cdots, Q_{t}^{\prime \prime}, r+1=s+t$, be the complementary set to the $Q_{k}^{\prime}$. Then summing independently over each $k_{j}^{\prime \prime}(j=1,2, \cdots, t)$ the equations $N\left[V\left(k_{0}, \cdots, k_{r}\right)\right]=N\left[W\left(k_{0}, \cdots, k_{r}\right)\right]$ we obtain:

$$
N\left[V\left(k_{1}^{\prime}, \cdots, k_{s}^{\prime}\right)\right]=N\left[W\left(k_{1}^{\prime}, \cdots, k_{s}^{\prime}\right)\right]
$$

where $V\left(k_{1}^{\prime}, \cdots, k_{s}^{\prime}\right)$ is the set of all $x \in L$ that are denominators of precisely $k_{i}^{\prime}$ members of $Q_{i}^{\prime}(i=1, \cdots, s)$ and of an arbitrary number of members of each $Q_{j}^{\prime \prime}$ in $x^{*} / x$, and where $W\left(k_{1}^{\prime}, \cdots, k_{s}^{\prime}\right)$ is defined dually.

Corollary 6.3.1. The number of elements of a finite modular lattice $L$ covered by precisely $k$ elements is equal to the number of elements of $L$ covering precisely $k$ elements.

Proof. First we note $v$ covers $x$ if and only if $v / x$ is a minimal prime quotient in $x^{*} / x$ (from some set of projective prime quotients) and dually. In Theorem 6.3 let $Q_{1}^{\prime}, \cdots, Q_{s}^{\prime}$ constitute all of the sets of projective prime quotients. Then summing over all sets of values of the $k_{i}^{\prime}$ for which $k_{1}^{\prime}+\cdots+k_{s}^{\prime}=k$ we obtain

$$
\sum_{k_{1}^{\prime}+\cdots+k_{s^{\prime}=k}} N\left[V\left(k_{1}^{\prime}, \cdots, k_{s}^{\prime}\right)\right]=\sum_{k_{1}^{\prime}+\cdots+k_{s^{\prime}=k}} N\left[W\left(k_{1}^{\prime}, \cdots, k_{s}^{\prime}\right)\right]
$$

This is the desired result.

Corollary 6.3.1 above is Dilworth's principal result in [3].

Corollary 6.3.2 (Same as Corollary 6.2.2). The number of meet irreducibles of a finite modular lattice is equal to the number of join irreducibles.

Alternative proof. Take $k=1$ in Corollary 6.3.1, noting by Theorem 1.1 that $x \in L$ is a meet irreducible if and only if $x$ is covered by precisely 1 element and dually.

Taking $s=1$ in Theorem 6.3 we obtain

Corollary 6.3.3. The number of $x \in L$, a finite modular lattice, that are the denominators of precisely $k$ members of a complete set $Q$ of projective complemented quotients of $L$ is equal to the number of $y \in L$ that are numerators of precisely $k$ members of $Q$.

7. The distributive case. We now make brief observations of our results as they appear in a distributive lattice $L$. The quotients $x^{*} / x$ and correspond- 
ing $y / y_{*}$ are complemented distributive lattices, therefore Boolean algebras. No pair of ideals of $x^{*} / x$ are projective in $L$ and dually no pair of dual ideals of $y / y_{*}$ are projective. Hence each $k_{i}=1$ or 0 in all nonempty $V\left(k_{0}, \cdots, k_{r}\right)$ and $W\left(k_{0}, \cdots, k_{r}\right)$. As observed in Theorem 1.5 each complete set $Q$ of projective quotient Boolean algebras has a unique maximal and unique minimal member. Hence the corresponding $V$ and $W$ sets of Theorems 6.1 and 6.2 consist of one element each $: x \in V$ and $y \in W$. These are the uniquely corresponding pairs of elements described in Theorem 1.5. When $Q$ is a set of projective prime quotients, $x \in V$ and corresponding $y \in W$ are a pair of uniquely corresponding meet and join irreducibles described in Theorem 1.2.

\section{REFERENCES}

1. G. Birkhoff, Lattice theory, rev. ed., Amer. Math. Soc. Colloquium Publications, vol. 25, 1948.

2. R. D. Carmichael, Introduction to the theory of groups of finite order, Boston, 1937.

3. R. P. Dilworth, Proof of a conjecture on finite modular lattices, Ann. of Math. vol. 60 (1954) pp. 359-364.

4. O. Veblen and W. H. Bussey, Finite projective geometries, Trans. Amer. Math. Soc. vol. 7 (1906) pp. 241-259.

5. L. Weisner, Abstract theory of inversion of finite series, Trans. Amer. Math. Soc. vol. 38 (1935) pp. 474-484.

UNIVERSITY OF WASHINGTON, Seattle, Wash. 